\title{
Measurement of Vacuum Pressure with a Magneto-Optical Trap: a Pressure-Rise Method
}

\author{
Rowan W. G. Moore, ${ }^{1}$ Lucie A. Lee, ${ }^{1}$ Elizabeth A. Findlay, ${ }^{1}$ Lara Torralbo-Campo, ${ }^{1}$ Graham D. Bruce, ${ }^{1}$ and \\ Donatella Cassettari ${ }^{1,}$ a) \\ SUPA School of Physics and Astronomy, University of St Andrews, North Haugh, St Andrews, Fife KY16 $9 S S$, \\ United Kingdom
}

(Dated: 9 July 2015)

The lifetime of an atom trap is often limited by the presence of residual background gases in the vacuum chamber. This leads to the lifetime being inversely proportional to the pressure. Here we use this dependence to estimate the pressure and to obtain pressure rate-of-rise curves, which are commonly used in vacuum science to evaluate the performance of a system. We observe different rates of pressure increase in response to different levels of outgassing in our system. Therefore we suggest that this is a sensitive method which will find useful applications in cold atom systems, in particular where the inclusion of a standard vacuum gauge is impractical.

There is a trend of making cold atom experiments simpler and more portable in view of taking them outside the laboratory ${ }^{1-8}$, where they can be used for applications such as precise inertial sensors ${ }^{9-13}$. In a compact apparatus, it is not always practical to include a vacuum gauge, and therefore alternative methods of estimating the background pressure are desirable. Given that pressure is in many cases the dominant factor affecting the lifetime of a trapped sample, the lifetime can in turn be used to estimate the pressure, effectively using the atom trap as a vacuum gauge.

In Ref. 14 this idea was developed into a quantitative method, which we further extend in the present paper by using a Magneto-Optical Trap (MOT) to acquire pressure rate-of-rise curves. These are useful diagnostic tools in vacuum science, and they are taken by turning off the pump after the base pressure of the system has been achieved and monitoring the subsequent pressure increase. The pressure evolution then indicates whether a leak is present, as well as measuring outgassing levels within the vacuum chamber. The latter are important in order to evaluate the outgassing behaviour of materials and components under vacuum, and to establish whether contamination is present. While pressure-rise curves are commonly measured with a vacuum gauge, in this paper we take the approach of using the effect of the pressure increase on the MOT. This offers the further advantage that the pressure is measured locally, rather than at a separate location of the vacuum system where the pressure may significantly differ due to limited conductance.

Our experiment is a vapour cell ${ }^{87} \mathrm{Rb}$ MOT. Because the MOT selectively loads rubidium atoms, but loses atoms to collisions with untrapped fast rubidium atoms and with other background gases, MOT measurements can be used to extract two distinct contributions to the pressure: that of the rubidium vapour, and that of any other background gas. To separate these contributions,

\footnotetext{
a)dc43@st-andrews.ac.uk
}

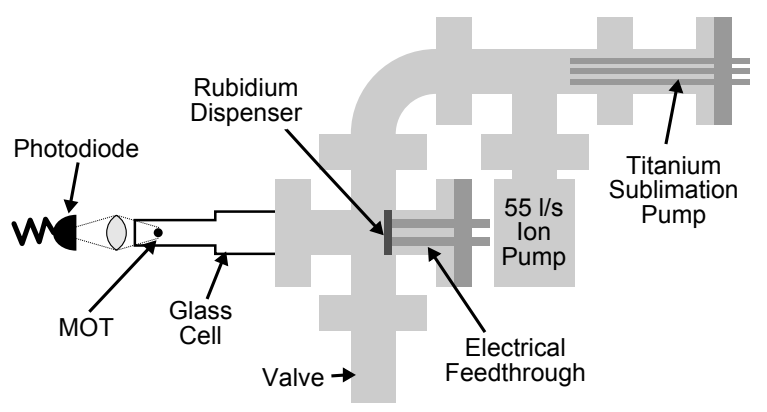

FIG. 1. Vacuum system: the MOT is created in the glass cell and the trapped atoms are monitored by collecting their fluorescence on a photodiode.

we first characterise our MOT at base pressure (i.e. with pumps on) by using an $N_{\text {eq }}-\tau$ plot: we acquire MOT loading curves and measure the equilibrium number of atoms $N_{\text {eq }}$ and the $1 / e$ loading time $\tau$. By repeating these measurements for different levels of rubidium pressure, we gain information on three parameters that characterise the MOT: the trapping cross section, the loss rate due to collisions with non-Rb background gases, and the lossrate coefficient for the collisions with $\mathrm{Rb}$ background. These measurements fully characterise our MOT. To acquire pressure-rise curves, we then turn off the ion pump and monitor the MOT over a period of hours, while the pressure in the system slowly rises. The MOT parameters determined from the initial characterisation are then used to convert these data into quantitative evolutions of the $\mathrm{Rb}$ pressure and of the non- $\mathrm{Rb}$ pressure.

Our six-beam MOT is created in a pyrex cell with $30 \mathrm{~mW}$ of optical power and a magnetic field gradient of $18 \mathrm{G} / \mathrm{cm}$. The trapped atoms are detected by collecting fluorescence with a photodiode. The vacuum system is shown in Fig. 1 and also comprises an isotopically pure ${ }^{87} \mathrm{Rb}$ dispenser from Alvatec, a $55 \mathrm{~L} / \mathrm{s}$ ion pump and a titanium sublimation pump. After assembly, the system was baked at $220^{\circ} \mathrm{C}$ and a base pressure of the order of $2 \times 10^{-10}$ Torr was obtained in the ion pump region. 

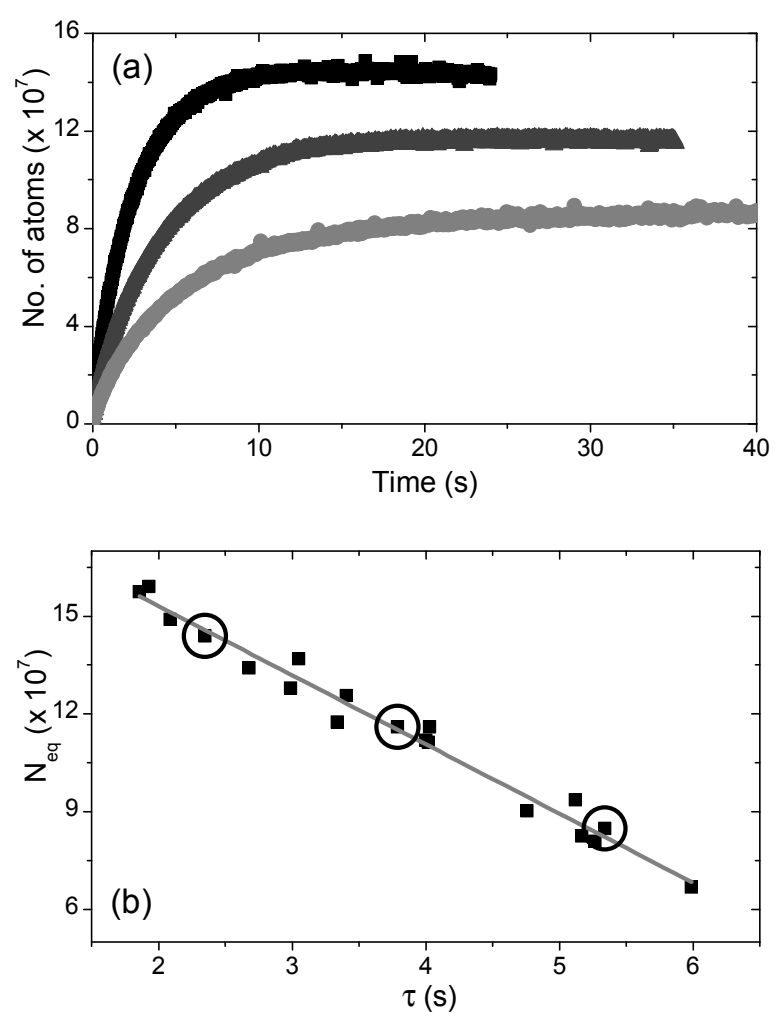

FIG. 2. Construction of the $N_{\text {eq }}-\tau$ plot with (a) the MOT loading with different levels of $\mathrm{Rb}$ pressure, and (b) the resultant $N_{\text {eq }}-\tau$ plot where the data shown in (a) have been encircled. The data in (b) are fitted with (5).

For a MOT loaded from background vapour, the MOT dynamics can be well approximated by the following rate equation $^{14,15}$ :

$$
\frac{d N(t)}{d t}=\alpha P_{\mathrm{Rb}}-\left(\beta P_{\mathrm{Rb}}+\gamma\right) N(t) .
$$

This describes the balance between the rates at which atoms are added to and lost from the trapped population $N$. The first term on the right-hand side is the rate at which atoms are captured; the constant $\alpha$ represents the MOT trapping cross-section while $P_{\mathrm{Rb}}$ is the partial $\mathrm{Rb}$ pressure. The second set of terms represents the losses from the trap. The first of these terms, $\beta P_{\mathrm{Rb}} N$, describes losses due to collisions with background $\mathrm{Rb}$ atoms. The second term, $\gamma N$, describes losses due to collisions with non-Rb background. Here we do not include the loss term due to two-body inelastic collisions between cold trapped atoms. Because our MOTs are always large enough to be in the constant-density limit, this loss rate is constant. Ref. 14 gives estimates of this loss rate, for MOTs similar to ours, which are well below our value for $\gamma$. Therefore we omit two-body losses from our discussion. We also note that in the pressure rise curves shown below, a nonnegligible two-body loss term would only introduce an offset in the conversion from loss rate to non-Rb pressure, hence it would not affect the measured rates of pressure increase.

The solution of (1) is

$$
N(t)=N_{\text {eq }}(1-\exp (-t / \tau)),
$$

where the equilibrium number of atoms in the MOT is

$$
N_{\mathrm{eq}}=\alpha P_{\mathrm{Rb}} \tau
$$

and the MOT loading time is

$$
\tau=1 /\left(\beta P_{\mathrm{Rb}}+\gamma\right)
$$

which coincides with the trap lifetime ${ }^{14}$. Combining (3) and (4) eliminates $P_{\mathrm{Rb}}$, giving

$$
N_{\mathrm{eq}}=\frac{\alpha}{\beta}(1-\gamma \tau)
$$

which relates the two easily measurable quantities $N_{\text {eq }}$ and $\tau$. Plotting (5) experimentally provides the initial characterisation of the MOT. For this purpose, a large amount of rubidium is released into the chamber, after which the Rb source is switched off. A sequence of loading curves is taken as the Rb partial pressure gradually decays, while the non-Rb partial pressure remains constant. This is continued until a data set spanning a sufficiently large range of $N_{\text {eq }}$ and $\tau$ is obtained as shown in Fig. 2. Fitting these data with (5) gives $\gamma=(0.11 \pm$ $0.01) \mathrm{s}^{-1}$ and $\alpha / \beta=(19.6 \pm 0.3) \times 10^{7}$.

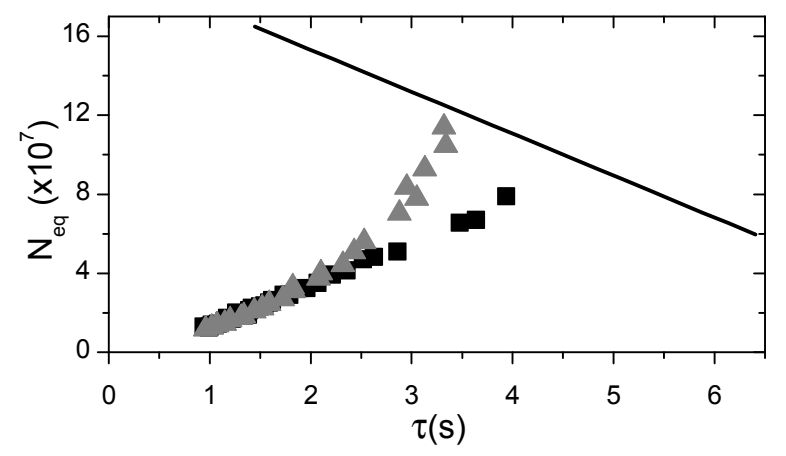

FIG. 3. The $N_{\text {eq }}-\tau$ plots measured with the dispenser current reduced to $4 \mathrm{~A}$ and the ion pump turned off. The solid line is the original $N_{\mathrm{eq}}-\tau$ plot from Fig. 2(b). The square and triangular data points are new $N_{\text {eq }}-\tau$ plots obtained after the ion pump has been turned off, starting at two different $N_{e q}$ values.

Physically, the value of $\alpha / \beta$ represents the largest MOT that can be obtained in our system, while $1 / \gamma$ is the theoretical upper limit for the loading time as the $\mathrm{Rb}$ pressure tends to zero, i.e. the longest possible trap lifetime in our system. This is a useful technique for MOT characterisation that we have previously applied to the study of MOT loading enhanced by Rb pressure modulation ${ }^{16}$. In the following this method is further applied to measuring pressure-rise curves in our system to 

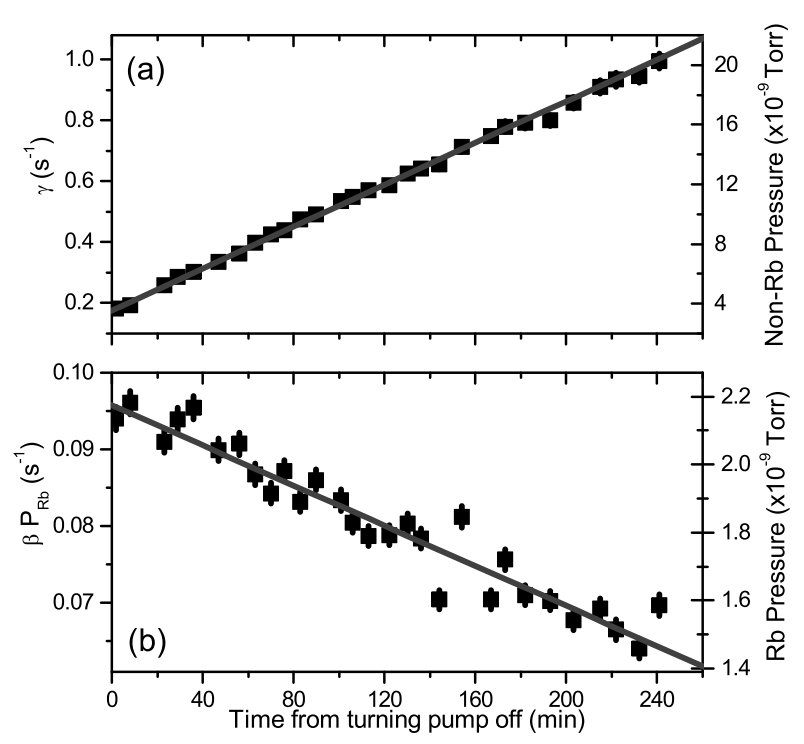

FIG. 4. Pressure evolution as a function of time after the ion pump has been switched off, extracted from the square data points in Fig. 3. (a) The non-Rb gases in the system show the expected pressure rise. The linear fit allows the determination of the gas load. (b) The Rb pressure decreases because the dispenser current has been lowered at $t=0$.

measure vacuum quality and distinguish between different levels of outgassing.

The value of $\gamma$ taken from the linear fit is directly proportional to the non-Rb pressure $P$ in the system. To convert $\gamma$ to pressure, we use $\gamma / P=4.9 \times 10^{7}$ Torr $^{-1} \mathrm{~s}^{-1}$ given in Ref. 14. This conversion factor is shown to vary by approximately a factor 2 depending on background gas composition, which is often an acceptable uncertainty for vacuum diagnostic purposes. Combined with $\gamma=0.11$ $\mathrm{s}^{-1}$ as obtained from Fig. 2, we estimate a base pressure of $2.2 \times 10^{-9}$ Torr. This estimate is higher than the value quoted above and the discrepancy can be explained by the limited conductance in our system.

The partial Rb pressure may also be calculated by using the conversion factor $\beta=4.4 \times 10^{7} \mathrm{Torr}^{-1} \mathrm{~s}^{-1}$ as given in Ref. 14. Thus $\alpha$ can be determined, and hence the rubidium pressure $P_{\mathrm{Rb}}=N_{\text {eq }} /(\alpha \tau)$ (using (3)). This pressure varies over the course of the measurements but a typical value is in the $10^{-9}$ Torr regime.

To obtain pressure-rise curves the procedure is similar to that for MOT characterisation, but with the ion pump switched off at an initial time $t=0$ to allow the non-Rb pressure to rise. Before $t=0$ the dispenser current is set at 5-6 A to trap large numbers of atoms in the MOT, and then is lowered to $4 \mathrm{~A}$ at $t=0$ to lower the $\mathrm{Rb}$ pressure and prevent overloading the chamber. MOT-loading curves are taken for up to four hours and $N_{\text {eq }}$ is again plotted as a function of $\tau$ as shown in Fig. 3. Both $N_{e q}$ and $\tau$ decrease over time as the quality of the vacuum deteriorates. This measurement is shown twice, starting from different initial rubidium pressures, and the

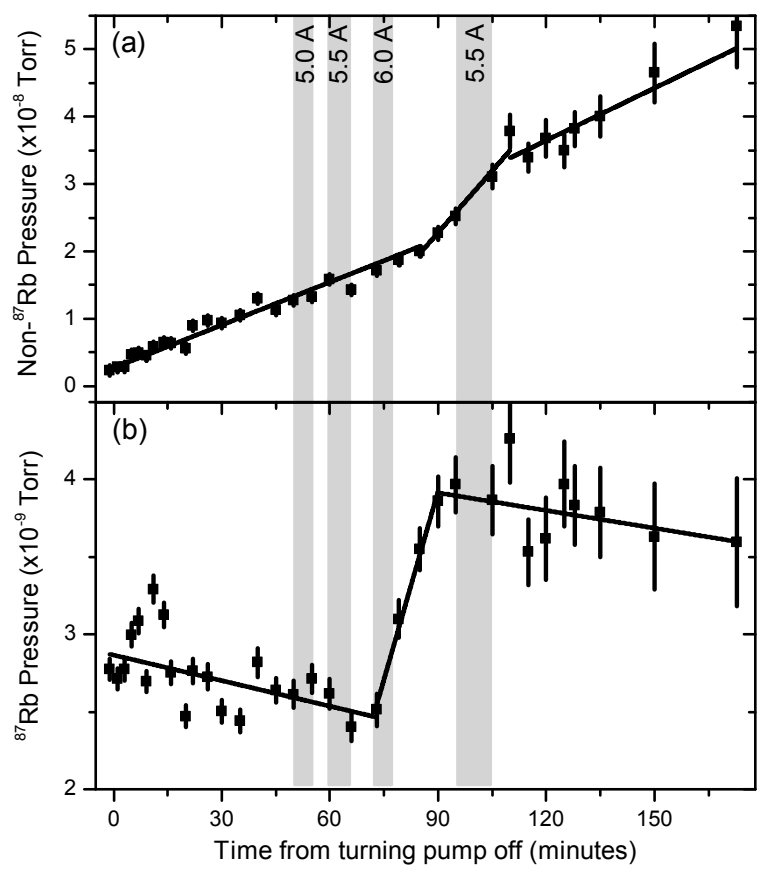

FIG. 5. Pressure evolution after turning off the ion pump, with pulses applied to the dispenser current. The vertical lines show the duration and current of each pulse. (a) Pressure rise of the non-Rb background showing temporary outgassing. (b) Evolution of the Rb pressure showing a clear increase after the 6 A pulse.

separate evolutions of $N_{\text {eq }}(\tau)$ are shown to converge.

Using $\alpha / \beta$ obtained from Fig. 2, the value of $\gamma$ for each time $t$ is calculated by rearranging (5):

$$
\gamma=\frac{1}{\tau}\left(1-\frac{\beta N_{\mathrm{eq}}}{\alpha}\right) .
$$

Once again, $\gamma$ is converted to pressure and this pressure is plotted as a function of time in Fig. 4(a), giving a pressure rise curve which is linear in time. From the measured rate of rise and the volume of the chamber $(\sim 1 \mathrm{~L})$, we estimate a gas load of $(1.10 \pm 0.02) \times 10^{-12}$ Torr L/s. We obtain comparable values for the gas load from both the square and the triangular data points in Fig. 3, confirming the robustness of this method. (This is in spite of the pronounced nonlinearity in the square dataset in Fig. 3 , which only arises from the larger change in dispenser current at $t=0$.) We take this gas load as a baseline for the subsequent comparative measurements of outgas rates, and note that this gas load is very low. By comparison, previously baked stainless steel (which constitutes most of the surface in our system) outgasses at a rate of $10^{-12}$ Torr L/ $/ \mathrm{s} \mathrm{cm}^{2}$, which corresponds to $>10^{-10}$ Torr $\mathrm{L} / \mathrm{s}$ for the surface of our system ${ }^{17}$. We attribute the observed low rate of pressure rise to the presence of an active titanium layer pumping gas in our system. Under these circumstances, the measured gas load is the partial contribution from gas species that are not pumped by the titanium layer. In our system this could be argon, which 
is present during the activation of the Alvatec dispenser. With regard to gases that are efficiently pumped by the titanium layer, for instance hydrogen, when the ion pump is switched off we expect them to quickly reach a new equilibrium pressure. This is established faster than the time resolution of our measurements, so that these gases do not contribute to the measured pressure-rise curves.

Our method is capable of discriminating between $\mathrm{Rb}$ and non- $\mathrm{Rb}$ pressure. The partial $\mathrm{Rb}$ pressure is plotted as a function of time in Fig. 4(b), using the conversion outlined above. Due to the reduction in dispenser current at $t=0$, the $\mathrm{Rb}$ pressure actually falls over time while the non- $\mathrm{Rb}$ pressure is rising. Rubidium pumping is always dominated by high adsorption to the steel walls of the vacuum chamber ${ }^{18}$ and therefore switching off the ion pump has little effect on the Rb pressure.

To test the sensitivity of our method to outgassing, we investigate the effect of repeatedly pulsing the dispenser to higher currents, as shown in Fig. 5. The current is kept at $4 \mathrm{~A}$ between pulses. After the $6 \mathrm{~A}$ pulse the $\mathrm{Rb}$ pressure clearly increases. However we also see that there is a temporarily increased rise in non- $\mathrm{Rb}$ pressure, i.e. an increased gas load. The rate of pressure rise then returns to the pre-pulse level which is comparable to the base gas load shown in Figure 4(a). This is indicative of a temporary outgassing either from within the dispenser, or from a region of the chamber that is being heated up by proximity to the dispenser. By measuring the rate increase in Fig. 5(a), we estimate a gas load from the 6 A pulse of $(6.7 \pm 1.1) \times 10^{-12}$ Torr $\mathrm{L} / \mathrm{s}$, which is small and compatible with UHV operation. The error in the measured rates determines the smallest outgassing rate which would be detectable here. This is of the order of $1 \times 10^{-12}$ Torr L/s, which is comparable to the sensitivity of standard helium leak testers.

In conclusion, we have used an $N_{\text {eq }}-\tau$ plot to characterise our MOT, and used the MOT effectively as a vacuum gauge to acquire pressure-rise curves, which quantify outgassing in our vacuum system. The small changes in gas load that we have detected demonstrate the sensitivity of the method. In the present work the measured gas load is only the partial contribution from gas not pumped by the titanium layer. If there was no titanium present, we would expect to measure the total gas load with similar sensitivity. It should also be possible to use this approach to check for leaks in a system and to discriminate between real and virtual leaks.

One particular advantage that this method has over a standard vacuum gauge is that the pressure is measured directly in the MOT region; this is more relevant for cold atoms experiments than the pressure at another point in the system. Moreover, we are able to separate the contribution to the pressure of the rubidium background, which can be monitored for the purpose of characterising the dispenser output. We expect this method to find broad applicability to cold atom experiments and to be of particular interest for applications that require a miniaturised vacuum system.

\section{ACKNOWLEDGMENTS}

R.M. and L.L. contributed equally to this work. We thank Robert Nyman for useful discussions and the anonymous reviewers for their helpful insights. This research was supported by UK EPSRC grant GR/T08272/01, IOP Scotland and the Leverhulme Trust Research Project Grant RPG-2013-074.

${ }^{1}$ S. Knappe, P. D. D. Schwindt, V. Gerginov, V. Shah, L. Liew, J. Moreland, H. G. Robinson, L. Hollberg, and J. Kitching, J. Opt. A: Pure Appl. Opt. 8, S318 (2006).

${ }^{2}$ F. Sorrentino, K. Bongs, P. Bouyer, L. Cacciapuoti, M. de Angelis, H. Dittus, W. Ertmer, A. Giorgini, J. Hartwig, M. Hauth, S. Herrmann, M. Inguscio, E. Kajari, T. T. Könemann, C. Lämmerzahl, A. Landragin, G. Modugno, F. Pereira dos Santos, A. Peters, M. Prevedelli, E. Rasel, W. Schleich, M. Schmidt, A. Senger, K. Sengstock, G. Stern, G. Tino, and R. Walser, Microgravity Sci. Technol. 22, 551 (2010).

${ }^{3}$ E. A. Salim, J. DeNatale, D. M. Farkas, K. M. Hudek, S. E. McBride, J. Michalchuk, R. Mihailovich, and D. Z. Anderson, Quantum Inf. Process. 10, 975 (2011).

${ }^{4}$ M. Schmidt, A. Senger, M. Hauth, C. Freier, V. Schkolnik, and A. Peters, Gyrosc. Navig. 2, 170 (2011).

${ }^{5}$ M. de Angelis, M. Angonin, Q. Beaufils, C. Becker, A. Bertoldi, K. Bongs, T. Bourdel, P. Bouyer, V. Boyer, S. Dörscher, H. Duncker, W. Ertmer, T. Fernholz, T. M. Fromhold, W. Herr, P. Krüger, C. Kürbis, C. Mellor, F. P. D. Santos, A. Peters, N. Poli, M. Popp, M. Prevedelli, E. Rasel, J. Rudolph, F. Schreck, K. Sengstock, F. Sorrentino, S. Stellmer, G. Tino, T. Valenzuela, T. Wendrich, A. Wicht, P. Windpassinger, and P. Wolf, Procedia Comput. Sci. 7, 334 (2011).

${ }^{6}$ B. Barrett, P.-A. Gominet, E. Cantin, L. Antoni-Micollier, A. Bertoldi, B. Battelier, P. Bouyer, J. Lautier, and A. Landragin, in Proceedings of the International School of Physics "Enrico Fermi", Vol. 188: Atom Interferometry (IOS Press, 2014) pp. 493-555.

${ }^{7}$ T. Farah, C. Guerlin, A. Landragin, P. Bouyer, S. Gaffet, F. Pereira Dos Santos, and S. Merlet, Gyrosc. Navig. 5, 266 (2014).

${ }^{8}$ J. Rushton, M. Aldous, and M. Himsworth, "The Feasibility of a Fully Miniaturized Magneto-Optical Trap for Portable Ultracold Quantum Technology," (2014), http://arxiv.org/abs/ 1405.3148.

${ }^{9}$ J. P. Dowling and G. J. Milburn, Phil. Trans. R. Soc. Lond. A 361, 1655 (2003).

${ }^{10}$ S. Bize, P. Laurent, M. Abgrall, H. Marion, I. Maksimovic, L. Cacciapuoti, J. Grünert, C. Vian, F. Pereira dos Santos, P. Rosenbusch, P. Lemonde, G. Santarelli, P. Wolf, A. Clairon, A. Luiten, M. Tobar, and C. Salomon, J. Phys. B: At. Mol. Opt. Phys. 38, S449 (2005).

${ }^{11}$ D. Budker and M. Romalis, Nat. Phys. 3, 227 (2007).

${ }^{12}$ A. D. Cronin, J. Schmiedmayer, and D. E. Pritchard, Rev. Mod. Phys. 81, 1051 (2009).

${ }^{13}$ J. Kitching, S. Knappe, and E. A. Donley, IEEE Sensors Journal 11, 1749 (2011).

${ }^{14}$ T. Arpornthip, C. A. Sackett, and K. J. Hughes, Phys. Rev. A 85, 033420 (2012).

${ }^{15}$ A. M. Steane, M. Chowdhury, and C. J. Foot, J. Opt. Soc. Am. B 9, 2142 (1992).

${ }^{16}$ L. Torralbo-Campo, G. D. Bruce, G. Smirne, and D. Cassettari, "Light-induced atomic desorption in a compact system for ultracold atoms," (2014), submitted to Phys. Rev. A http: //arxiv.org/abs/1312.6442.

${ }^{17}$ N. Yoshimura, Vacuum Technology (Chapman and Hall, Tokyo, 2008).

${ }^{18}$ C. Wieman, G. Flowers, and S. Gilbert, Am. J. Phys. 63, 317 (1995). 